\title{
A Novel Image Segmentation Algorithm Based on Fuzzy C-means Algorithm and Neutrosophic Set
}

\author{
Yanhui Guo ${ }^{1,2}$, H.D. Cheng ${ }^{1,2}$, Wei Zhao ${ }^{1}$, Yingtao Zhang ${ }^{1}$ \\ ${ }^{1}$ School of Computer Science and technology, Harbin Institute of Technology, Harbin, \\ China, 150001 \\ ${ }^{2}$ Department of Computer Science, Utah State University, Logan, UT 84322 U.S.A.
}

\begin{abstract}
Image segment is an important step in image processing, pattern recognition and computer vision. Numerous algorithms have been proposed to in this field for last twenty years. However, a generalized segmentation method, especial for noisy image, are not studied greatly.

A neutrosophic set (NS), a part of neutrosophy theory, studies the origin, nature, and scope of neutralities, as well as their interactions with different ideational spectra. The neutrosophic set is a formal framework that has been recently proposed. However, the neutrosophic set needs to be specified from a technical point of view for a given application or field. We apply the neutrosophic set in image domain and define some concepts and operations for image segmentation.

The image G is transformed into NS domain. Then, the entropy in neutrosophic set is defined and employed to evaluate the indeterminancy. A new operation, mean operation is proposed to reduce the set indeterminancy. Finally, a new fuzzy c-means algorithm, $\alpha$-fuzzy-c-means ( $\alpha$ FCM) is proposed to segment the image on NS domain. We have conducted experiments on a variety of images. The experimental results demonstrate that the proposed approach can segment the images automatically and effectively. Especially, it can process the "clean" images
\end{abstract}

and the images with noise without knowing its type, which is the most difficult task for image segmentation.

Keywords: Image segmentation, fuzzy cmeans, Neutrsophic set, Entropy.

\section{Introduction}

Image segmentation is a critical and essential component and is one of the most difficult tasks in image processing and pattern recognition.

Gray image segmentation approaches are based on either discontinuity and/or homogeneity of gray level values in a region. The approach based on discontinuity tends to partition an image by detecting isolated points, lines and edges according to abrupt changes in gray levels. The approaches based on homogeneity include thresholding, clustering, region growing, and region splitting and merging [1].

The fuzzy theory has been employed into image segmentation, which retain more information from the original image than hard segmentation methods [2, 3].

Fuzzy c-means (FCM) [4,5]is a method of clustering which allows one piece of data to belong to two or more clusters, which is frequently used in pattern recognition. The fuzzy c-means algorithm could obtain segmentation results by fuzzy pixel classification. Unlike hard

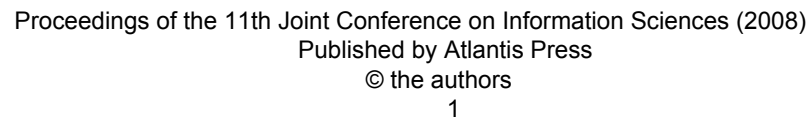


classification methods which force pixels to belong exclusively to one class, FCM allows pixels to belong to multiple classes with varying degrees of membership [6]. In general, the fuzzy C-means approach is highly effective for image segmentation.

Neutrosophy, a new branch of philosophy, as a generalization of dialectics, studies the origin, nature, and scope of neutralities, as well as their interactions with different ideational spectra [7]. Neutrosophy theory considers proposition, theory, event, concept, or entity, $<\mathrm{A}>$ in relation to its opposite, $<$ Anti-A $>$ and the neutrality $\langle$ Neut-A $>$, which is neither $<$ A $>$ nor $<$ Anti-A $>$. The $<$ Neut-A $>$ and $<$ Anti-A $>$ are referred to as $<$ Non-A $>$. According to this theory, every idea $<\mathrm{A}>$ tends to be neutralized and balanced by $<$ Anti-A $>$ and $<$ Non-A $>$.

Neutrosohic set provides a new powerful tool to describe the image with uncertainty information, which had been applied to image processing techniques, such as image thresholding. [8] proposed a thresholding algorithm based on neutrosophic, which could select the thresholds automatically and effectively.

In this paper, the new Neutrosphic set approach for image segmentation is proposed. The experiments on real images demonstrate that the proposed approach can perform segmentation well.

\section{Proposed method}

\subsection{Neutrosphic set}

Neutrosophic set and its properties are discussed briefly [7].

Definition 1 (Neutrosophic set) Let $U$ be a universe of discourse, and a neutrosophic set $A$ is included in $U$. An element $x$ in set $M$ is noted as $x(T, I, F) . T, I$ and $F$ are real standard or non-standard sets of $]^{-} 0,1^{+}[$

$$
\begin{aligned}
& \text { with } \sup T=t_{-} \text {sup , inf } T=t \_i n f \text {, } \\
& \sup I=i \_ \text {sup , } \quad \text { inf } I=i \_i n f \text {, } \\
& \sup F=f_{-} \text {sup }, \quad \text { inf } F=f_{-} \text {inf } \text { and } \\
& n \_ \text {sup }=t \_ \text {sup }+i_{-} \sup +f \_ \text {sup } \text {, } \\
& n \_ \text {inf }=t \_ \text {inf }+i_{-} \text {inf }+f \_ \text {inf } . T, I \text { and }
\end{aligned}
$$

\subsection{Neutrosophic image}

Definition 2 (Neutrosophic image): Let $U$ be a universe of the discourse, and $W \subseteq U$ which is composed by the bright pixels. A neutrosophic image $P_{N S}$ is characterized by three membership sets $T, I$ and $F$.

A pixel $P$ in the image is described as $P(t, i, f)$ and belongs to $W$ in the following way: it is $t \%$ true in the bright pixel set, $i \%$ indeterminate, and $f \%$ false, where $t$ varies in $T, i$ varies in $I$, and $f$ varies in $F$.

The pixel $P(i, j)$ in the image domain is transformed into the neutrosophic domain. $P_{N S}(i, j)=\{T(i, j), I(i, j), F(i, j)\}$. $T(i, j), I(i, j)$ and $F(i, j)$ are the membership value belonging to white set, indeterminate set and non-white set, respectively, which are defined as:

$$
\begin{gathered}
T(i, j)=\frac{\bar{g}(i, j)-\bar{g}_{\min }}{\bar{g}_{\max }-\bar{g}_{\min }} \\
\bar{g}(i, j)=\frac{1}{w \times w} \sum_{m=i-w / 2}^{i+w / 2} \sum_{n=j-w / 2}^{j+w / 2} g(m, n) \\
I(i, j)=\frac{\delta(i, j)-\delta_{\min }}{\delta_{\max }-\delta_{\min }}(3) \\
\delta(i, j)=a b s(g(i, j)-\bar{g}(i, j)) \\
F(i, j)=1-T(i, j)(5)
\end{gathered}
$$

where $\bar{g}(i, j)$ is the local mean value of the image. $\delta(i, j)$ is the absolute value of the difference between intensity $g(i, j)$ and its local mean value $\bar{g}(i, j)$ at $(i, j)$.

\subsection{Neutrosophic Image Entropy}


Definition 3 (Neutrosophic image entropy) The neutrosphic image entropy is defined as the summation of the entropies of three sets $T, I$ and $F$, which is employed to evaluate the distribution of the elements in the neutrosphic domain:

$$
\begin{gathered}
E n_{N S}=E n_{T}+E n_{I}+E n_{F} \text { (6) } \\
E n_{T}=-\sum_{i=\min \{T\}}^{\max \{T\}} p_{T}(i) \ln p_{T}(i) \\
E n_{I}=-\sum_{i=\min \{I\}}^{\max \{I\}} p_{I}(i) \ln p_{I}(i)(8) \\
E n_{F}=-\sum_{i=\min \{F\}}^{\max \{F\}} p_{F}(i) \ln p_{F}(i)(9)
\end{gathered}
$$

where $E n_{T}, E n_{I}$ and $E n_{F}$ are the entropies of the sets $T, I$ and $F$, respectively. $p_{T}(i), p_{I}(i)$ and $p_{F}(i)$ are the probabilities of elements in $T, I$ and $F$, respectively, whose values equal to $i$.

\section{4. $\alpha$-mean operation}

Definition 4 ( $\alpha$-mean operation): The $\alpha$-mean operation for $P_{N S}, \bar{P}_{N S}(\alpha)$ is defined as:

$$
\begin{gathered}
\bar{P}_{N S}(\alpha)=P(\bar{T}(\alpha), \bar{I}(\alpha), \bar{F}(\alpha))(10) \\
\bar{T}(\alpha)=\left\{\begin{array}{cc}
T & I<\alpha \\
\bar{T}_{\alpha} & I \geq \alpha
\end{array}(11)\right. \\
\bar{T}_{\alpha}(i, j)=\frac{1}{w \times w}\left(\sum_{m=-i-w / 2 n=j-w / 2}^{i+w / 2} \sum^{j+w / 2} T(m, n)-T(i, j)\right) \\
\bar{F}(\alpha)=\left\{\begin{array}{ll}
F & I<\alpha \\
\bar{F} & I \geq \alpha
\end{array}(13)\right. \\
\bar{F}_{\alpha}(i, j)=\frac{1}{w \times w}\left(\sum_{m=-i w 2 n=j-w / 2}^{i+w / 2} \sum_{j+w / 2} F(m n)-F(i, j)\right) \\
\bar{I}_{\alpha}(i, j)=\frac{\bar{\delta}_{T}(i, j)-\bar{\delta}_{T \min }}{\bar{\delta}_{T \max }-\bar{\delta}_{T \min }}(14) \\
\bar{\delta}_{T}(i, j)=a b s(\bar{T}(i, j)-\overline{\bar{T}}(i, j))(16) \\
\overline{\bar{T}}(i, j)=\frac{1}{w \times W}\left(\sum_{m i-w / 2 n=j-w / 2}^{i+w / 2} \sum^{j+w / 2} \bar{T}(m n)-\bar{T}(i, j)\right)(17)
\end{gathered}
$$

where $\bar{\delta}_{T}(i, j)$ is the absolute value of the difference between the mean intensity $\bar{T}(i, j)$ and its mean value $\overline{\bar{T}}(i, j)$ after the $\alpha$-mean operation.

\section{5. $\alpha$-fuzzy-c-means algorithm}

A new clustering method is defined for the neutrosophic set and it deals with $\bar{P}_{N S}(\alpha)$, the neutrosophic set after the $\alpha$ mean operation.

Considering the effect of indeterminacy, we composed the two set, $T$ and $I$ into a new value for clustering.

$$
X(i, j)=\left\{\begin{array}{cc}
T(i, j) & I(i, j) \leq \alpha \\
\bar{T}_{\alpha}(i, j) & I(i, j)>\alpha
\end{array}\right.
$$

A new modified fuzzy c-mean algorithm on neutrosphic set is proposed. The new objective function is defined as:

$$
J_{N Q}=\sum_{l=1}^{C} \sum_{i=1}^{H} W\left(\mu_{j=1}^{W}(i, j)\right)^{m}\left\|X(i, j)-c_{1}\right\|^{2}
$$

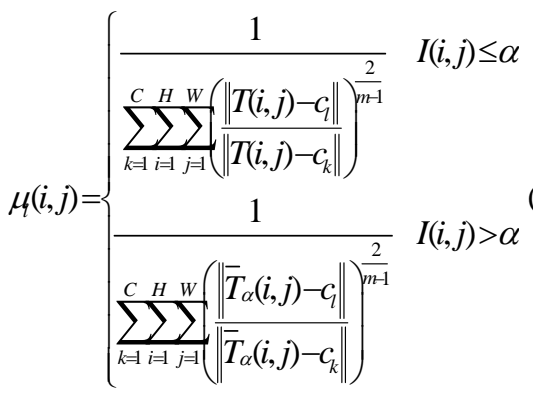

The algorithm is composed of the following steps:

1) Initialize the partition matrix ${\underset{\sim}{(0)}}^{(0)}$;

2) Calculate the centers vectors $C^{(k)}$ with ${\underset{\sim}{U}}^{(k)}$ at k step; 
$C_{l}= \begin{cases}\frac{\sum_{i=1}^{H} \sum_{j=1}^{W}\left(\mu_{4}(i, j)\right)^{m} \cdot T(i, j)}{\sum_{i=1}^{H} \sum_{j=1}^{W}\left(\mu_{4}(i, j)\right)^{m}} & I(i, j) \leq \alpha \\ \frac{\sum_{i=1}^{H} \sum_{j=1}^{W}\left(\mu_{l}(i, j)\right)^{m} \cdot \bar{T}_{\alpha}(i, j)}{\sum_{i=1}^{H} \sum_{j=1}^{W}\left(\mu_{4}(i, j)\right)^{m}} & I(i, j)>\alpha\end{cases}$

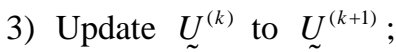

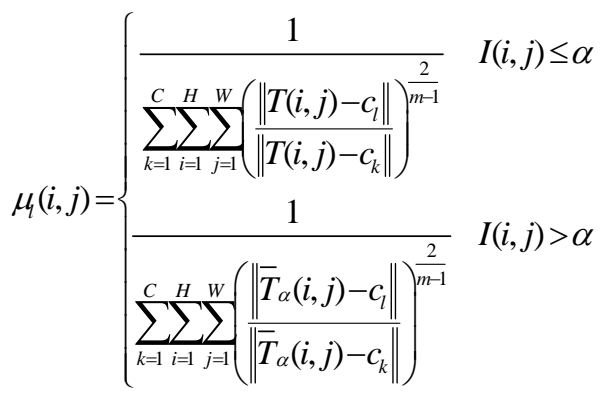

4) If $\max _{i, j, l}\left\{\left|\mu_{l}^{(k+1)}(i, j)-\mu_{l}^{(k)}(i, j)\right|\right\}<\varepsilon$

then stop;

Otherwise return to step 2).

\subsection{Image segmentation based on $\alpha$ - fuzzy-c-means}

A novel image segmentation algorithm, $\alpha$-fuzzy-c-mean algorithm, is proposed, which is composed of the following steps: Step 1: Input the image;

Step 2: Transform the image into NS domain;

Step 3: Perform the $\alpha$-mean operation on the subset $T$;

Step 4: Compute the entropy of the indeterminate subset $I, E n_{I}(i)$;

Step 5: If $\frac{E n_{I}(i+1)-E n_{I}(i)}{E n_{I}(i)}<\delta$, go to

Step 6; Else go to Step 3;

Step 6: Apply the $\alpha$-fuzzy-c-mean clustering on the neutrosophic set;

Step 7: Segment the image according to the result in step 6;

\section{Experiments and discussions}

We have applied the proposed approach to a variety of real images, and compared with a modified fuzzy C-means segmentation algorithm (MFCM) [9].

Fig, 1(a) is the Lena image. Fig. 5(b) is the segmented image using the MFCM algorithm with three classes. The regions on the hair and the hat are not homogenous and separated using the MFCM approach, which are caused by the noise and the textures on the hair and the hat. However, the AFCM approach can successfully partition the image into desired regions, as shown in the Fig. 5(c). The regions after segmentation in Fig.5(c) are more consistent and are not affected by the noise on the hair and the hat.

Fig. 2(a) has two pandas on the image, which has three main regions: the pandas, the stones and the grass. Fig. 2(b) is the result of the MFCM algorithm, which contains some misclassified regions, especially, in the grass region and stone region. In addition, the pandas' backs are affected by some noise pixels. In Fig. 6(c), the proposed approach makes the three regions distinctly and easy to recognize. The segmented result is more homogenous and easier to detect.

Fig. 3(a) is the Lena image with Guassian noise (mean is 0 and variance is 2.55). Fig. 3(b) is the segmentation result using the MFCM method, which is affected greatly by the noise, while the result by the proposed method is much better, as shown in Fig. 8(c), and the hat, hair and woman's face are segmented correctly.

In summary, the proposed method not only can segment the clear images, but also can segment noisy images, due to the fact that the proposed approach can handle the indeterminancy of the images well.

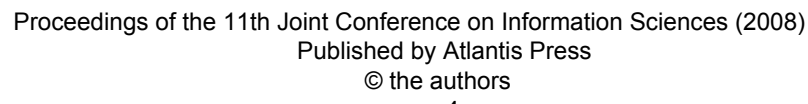




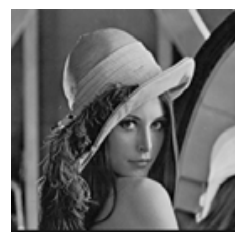

(a)

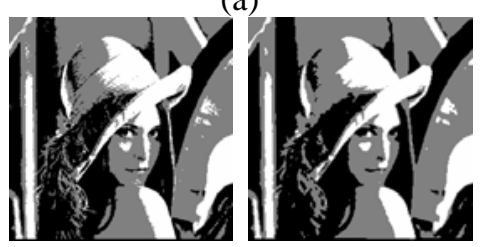

(b)

(c)

Fig. 1. Lena image.

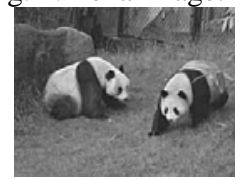

(a)

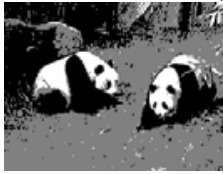

(b)

Fig. 2. Pandas image

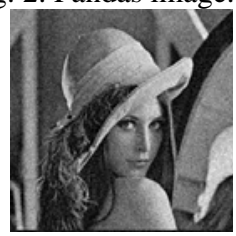

(a)

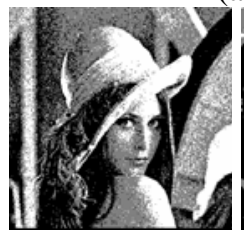

(b)

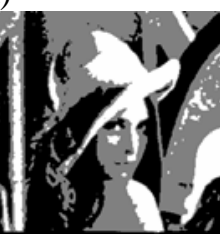

(c)

Fig. 3. Lena image with Gaussian noises.

\section{Conclusions}

In this paper, a novel neutrosophic set approach to image segmentation is proposed based on fuzzy c-means cluster analysis. The image is described using three membership sets, $T, F$ and $I$. The entropy in NS domain is defined and employed to evaluate the indeterminancy. A new operation in neutrosophic set domain, the $\alpha$-mean operation, is proposed to reduce the set's indeterminancy. The image becomes more uniform and homogenous after the $\alpha$-mean operation, and more suitable for segmentation. Finally, the image in NS domain is segmented using a new fuzzy c-means clustering method. The membership value in the fuzzy clustering frame is defined and updated according to the indeterminancy value in NS domain. The experimental results show that the proposed method can not only perform better on 'clean' images, but also on the noisy images. The proposed approach can find more applications in image processing and patter recognition.

\section{Acknowledgement}

The work was supported, in part, by Natural Scientific Research Innovation Foundation in Harbin Institute of Technology, Project HIT.NSRIF.2008.48, and Natural Science Foundation of China No.60873142 and No. 30670546.

\section{References}

[1] H. D. Cheng, X. H. Jiang, Y. Sun and J. Wang, "Color image segmentation: advances and prospects," Pattern Recognition, vol.34, pp. 2259-2281, 2001.

[2] J. C. Bezdek, L. O. Hall and L. P. Clarke, "Review of MR image segmentation techniques using pattern recognition," Medical Physics, vol.20, pp. 1033, 1993.

[3] J. K. Udupa and S. Samarasekera, "Fuzzy Connectedness and Object Definition: Theory, Algorithms, and Applications in Image Segmentation," Graphical Models and Image Processing, vol.58, pp. 246-261, 1996.

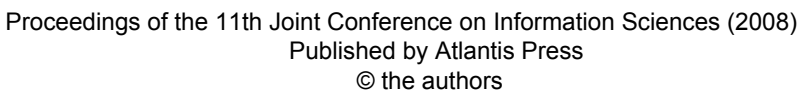


[4] J. C. Dunn, "A fuzzy relative of the ISODATA process and its use in detecting compact well-separated clusters," Journal of Cybernetics, vol.3, pp. 32-57, 1973.

[5] J. C. Bezdek, Pattern Recognition with Fuzzy Objective Function Algorithms, Kluwer Academic Publishers Norwell, MA, USA, 1981.

[6] D. L. Pham and J. L. Prince, "An adaptive fuzzy C-means algorithm for image segmentation in the presence of intensity inhomogeneities," Pattern Recognition Letters, vol.20, pp. 57-68, 1999.

[7] F. Smarandache, A Unifying Field in Logics Neutrosophic Logic. Neutro- sophy, Neutrosophic Set, Neutrosophic Probability, American Research Press, 2003.

[8] H. D. Cheng and Y. Guo, "A new neutrosophic appraoch to image thresholding," accepted by New Mathematics and Natural Computation, 2008.

[9] L. Ma and R. C. Staunton, "A modified fuzzy C-means image segmentation algorithm for use with uneven illumination patterns," Pattern Recognition, vol.40, pp. 3005-3011, 2007 